\title{
Nerol protects against hypoxia/reoxygenation-induced apoptotic injury by activating PI3KJAKT signaling in cardiomyocytes
}

\author{
Jin CHENG, Qing ZOU, Yugang XUE* \\ Department of Cardiology, Tangdu Hospital, Air force Military Medical University, Xi'an 710000, China. \\ *Correspondence: xueyugang123456@163.com \\ https://doi.org/10.37175/stemedicine.v2i6.87
}

\begin{abstract}
Background: Nerol was reported as a natural anti-oxidant product and its protective effects against cardiovascular diseases have been documented. Our current study was designed to explore the cardioprotective effect of Nerol on hypoxia/reoxygenation (H/R)-induced production of reactive oxygen species (ROS) and cell apoptosis in H9c2 cells. The potential molecular mechanisms were further investigated.

Methods: The cells were treated with 2.5 or $5 \mu \mathrm{M}$ Nerol before or after H/R. Lactate dehydrogenase (LDH) release, cell viability, oxidative stress markers, and apoptotic proteins were assessed by cell counting kit-8, LDH release assay, commercial kits, and Western blot, respectively. To explore the underlying mechanism, the phosphorylation of $\mathrm{p} 85$ and $\mathrm{p} 38$, regulatory subunits of phosphoinositide-3-kinase (PI3K)/ protein kinase B (AKT) and mitogen-activated protein kinase (MAPK), was evaluated by Western blot. To further confirm that the PI3K/AKT signaling pathway participated in the cardiomyocyte protection, H9c2 cells were treated with $5 \mu \mathrm{M}$ Nerol in the presence or absence of $5 \mu \mathrm{M}$ BEZ235 or LY294002 followed by $H / R$ treatment.
\end{abstract}

Results: H/R remarkably induced apoptosis, LDH release and ROS production. The cell viability was suppressed via inhibiting the PI3K/AKT signaling pathway activation. By contrast, pretreatment with Nerol can neutralize these effects by activating the PI3K/AKT signaling pathway. With the addition of BEZ235 or LY294002, the inhibitory effects of Nerol were abolished.

Conclusion: Nerol provided promising cardioprotective effect against $\mathrm{H} / \mathrm{R}$-induced injuries in H9c2 cells by activating the PI3K/AKT pathway.

Keywords: Nerol · Oxidative stress · Hypoxia/reoxygenation · Apoptosis · PI3K/AKT signaling

\section{Introduction}

The heart is a high oxygen consumption organ and the cardiomyocytes are very sensitive to oxygen deprivation (1). The reoxygenation following hypoxia normally causes massive production of reactive oxygen species (ROS), which results in oxidative damage in the pathophysiology of hypoxia/reoxygenation (H/R) injury (2). Overwhelming ROS production results in remarkable damage to

Received: Mar 12, 2021; Accepted: Mar 25, 2021

(c) The Author(s). 2020 This is an Open Access article distributed under the terms of the Creative Commons License (http://creativecommons.org/licenses/by/4.0/) which permits unrestricted use, distribution, and reproduction in any medium or format, provided the original work is properly cited. cardiomyocytes and impairs the balance between oxidation and antioxidation (3). Oxidative stress is one of the main contributions to apoptosis, thus alleviating and inhibiting ROS production may be a potential therapeutic approach to promote survival and inhibit apoptosis of cardiomyocytes (4)

Discovering and developing a novel natural product to prevent and treat cardiovascular diseases resulted from oxidative stress is very meaningful (5-7). The cardioprotective effects of grape seed proanthocyanidin extract against cardiomyocyte apoptosis, DNA damages and histopathological changes have been attributed to its superior antioxidant efficacy (6). Quercetin, a flavonoid anti-oxidant present in vegetables and fruits, also shows 
cardioprotective effects through its anti-oxidative stress property (7). Previous study has revealed the cardioprotective effects of Rosa Damascene petals against myocardial infarction in rats by restoring the cardiac marker enzymes and increasing myocardial anti-oxidants (8). Nerol, one of the main chemical compositions in Rosa damascene, is known as a common constituent of several essential oils (9). It has been demonstrated that Nerol in the essential oils promotes the superoxide scavenging activities (10). The anti-oxidant potential of Nerol attracts widespread interest (9). Nerol shows a promising anti-oxidant property in hydroperoxide-stressed rat alveolar macrophages, which promotes cell viability and inhibits ROS generation (11). After being pretreated with Nerol, the arrhythmia severity index can be significantly attenuated by reducing $\mathrm{Ca}^{2+}$ influx in guinea pig hearts (12). However, it is largely unknown concerning the cardioprotective effects of Nerol against H/R-induced injuries in cardiomyocytes.

\section{Materials and Methods \\ Cell culture and treatment}

H9c2 cells (a rat embryonic heart cardiomyocyte cell line) was acquired from the American Type Culture Collection (ATCC, Manassas, VA). The cells were cultured in Dulbecco's modified Eagle's medium (DMEM, Invitrogen), 10\% fetal bovine serum (FBS, Gibco), and $1 \%$ penicillin/streptomycin $(1 \%)$ at $37{ }^{\circ} \mathrm{C}$ in a humidified culture incubator $\left(5 \% \mathrm{CO}_{2}\right.$ and $95 \%$ air $)$.

\section{H/R model}

To evaluate the H/R-induced injury, H9c2 cells with or without Nerol treatment were cultured in serumand glucose-free medium and incubated under hypoxic conditions $\left(94 \% \mathrm{~N} 2,1 \% \mathrm{O}_{2}\right.$ and $5 \% \mathrm{CO}_{2}$ ) for $12 \mathrm{~h}$ at 37 ${ }^{\circ} \mathrm{C}$, and then cultured under reoxygenation conditions (5\% $\mathrm{CO}_{2}$ and $95 \%$ air) in complete medium for another $12 \mathrm{~h}$ at $37{ }^{\circ} \mathrm{C}$. The cells cultured under normoxic conditions were used as the control group. To determine the effects of Nerol on H/R-induced injuries, H9c2 cells were pretreated with 2.5 or $5 \mu \mathrm{M}$ Nerol (Figure 1A, Selleckchem, Houston, TX), followed by hypoxia (12 h) and reoxygenation $(12 \mathrm{~h})$. To determine the potential therapeutic role of Nerol in pathological cardiac patients, the H9c2 cells were treated with Nerol after hypoxia/ reoxygenation-induced injury. The corresponding lactate dehydrogenase $(\mathrm{LDH})$ release and cell viability were determined. To evaluate the effects of Nerol on H9c2 cells following the treatment of phosphoinositide-3kinase (PI3K) inhibitors, H9c2 cells were incubated with the serum- and glucose-free medium supplemented with Nerol $(5 \mu \mathrm{M})$ with or without BEZ235 (5 $\mu \mathrm{M}$, a dual PI3K/mTOR inhibitor, Sigma) or LY294002 (a strong PI3K inhibitor, Sigma), before H/R induction as aforementioned.

\section{Cell viability determination}

Cell counting kit-8 (CCK-8) assay was used to evaluate cell viability. Briefly, H9c2 cells were seeded to 96-well plates with $5 \times 10^{4}$ cells per well and treated with Nerol, followed by incubating with the CCK-8 reagent at $37{ }^{\circ} \mathrm{C}$ with $5 \% \mathrm{CO}_{2}$ for another $2 \mathrm{~h}$. A microplate reader was used to read the absorbance at a wavelength of $450 \mathrm{~nm}$.

\section{LDH release assay}

The LDH level was determined to evaluate the H/ R-induced cell necrosis according to the manufacture's instruction (Takara, Dalian, China). The cells were subjected distinct treatments as mentioned before. The supernatant was carefully collected after the microplate was centrifuged at $250 \mathrm{~g}$ for $10 \mathrm{~min}$. The supernatants were incubated with the reaction mixture for $30 \mathrm{~min}$ at room temperature. A microplate reader was used to read the absorbance at a wavelength of $490 \mathrm{~nm}$.

\section{Oxidative stress marker analysis}

The levels of glutathione peroxidase (GSP-Px), catalase (CAT), superoxide dismutase (SOD) and malondialdehyde (MDA) were measured by commercial assay kits (Beyotime, Shanghai, China). In brief, the cells were lysed and homogenized by $1 \%$ Nonidet P-40 (NP-40, SigmaAldrich) lysis buffer containing $20 \mathrm{mM}$ Tris, $137 \mathrm{mM}$ $\mathrm{NaCl}, 20 \%$ glycerol, $10 \mathrm{mM} \mathrm{NaF}, 1 \mathrm{mM} \mathrm{Na} \mathrm{VO}_{4}, 10 \mathrm{mM}$ phenylmethylsulfonyl fluoride, and $1 \times$ proteinase inhibitor cocktail, pH 7.4. The activity of CAT, GSH-Px and MDA in the homogenates was determined. To determine the activity of SOD, the cells were washed by ice-cold phosphatebuffered saline (PBS) and lysed by provided lysis buffer. The homogenate was centrifuged at 12,000 g for $5 \mathrm{~min}$ at $4{ }^{\circ} \mathrm{C}$. The supernatant was collected to determine the concentration of SOD.

\section{Cell apoptosis analysis}

A commercial Annexin V-FITC/propidium iodide (PI) apoptosis detection kit (Sigma-Aldrich) was used to analyze the H9c2 cells with different treatments as described above. The cells were trypsinized and washed with cold PBS, followed by resuspending in $1 \times$ binding buffer. H9c2 cells were further incubated with PI and FITC Annexin V for 13-15 min in the dark. The apoptotic cells were quantified by flow cytometry using a FACScan flow cytometry and CellQuestTM software (BD Biosciences, San Jose, CA, US). 
A<smiles>CC(C)=CCC/C(C)=C\CO</smiles>

Nerol

D

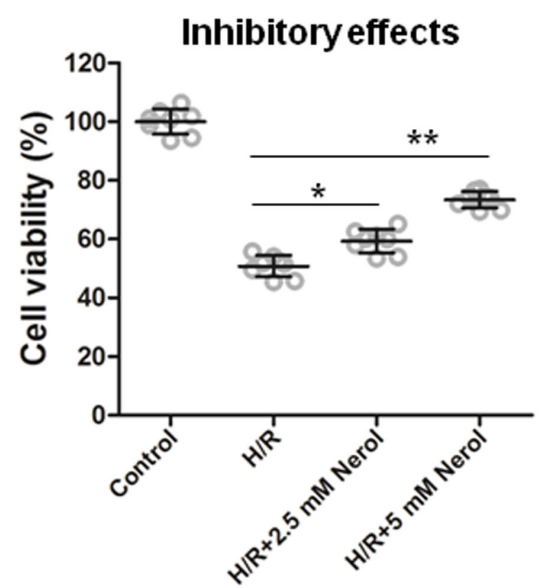

B

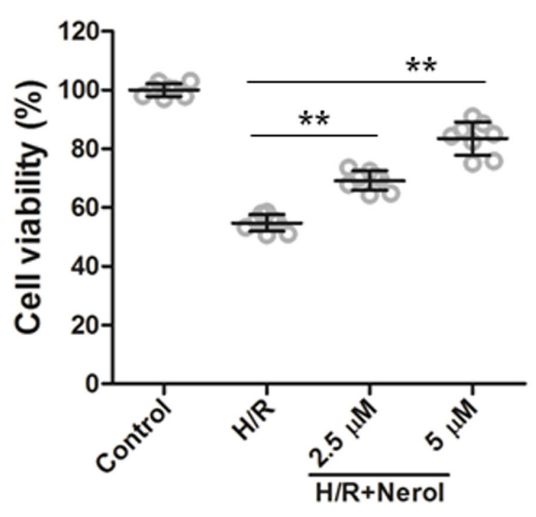

C

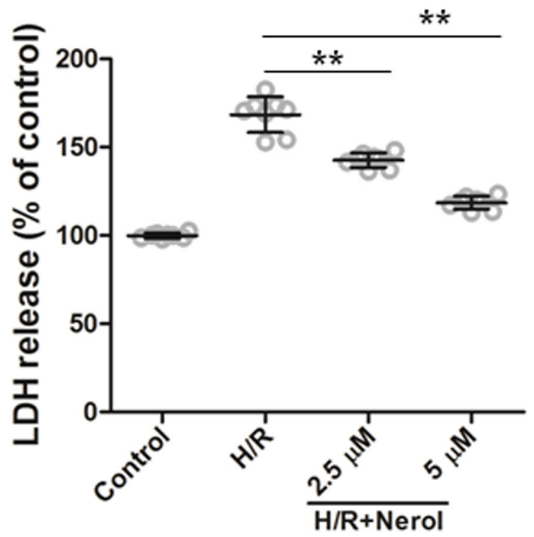

E

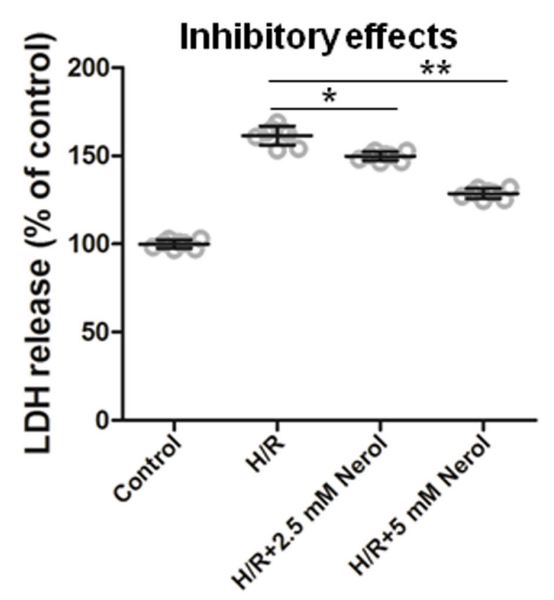

Figure 1. Nerol alleviated H/R-induced injuries in cardiomyocytes. (A) Chemical structure of Nerol. (B) H9c2 cardiomyocytes were treated with $2.5 \mu \mathrm{M}$ or $5 \mu \mathrm{M}$ Nerol, followed by H/R treatment for $12 / 12 \mathrm{~h}$, followed by CCK-8 assay. (C) H9c2 cardiomyocytes were treated with $2.5 \mu \mathrm{M}$ or $5 \mu \mathrm{M}$ Nerol, followed by H/R treatment for 12/12 h, followed by LDH release assay. (D) H9c2 cardiomyocytes were pre-treated with H/ $\mathrm{R}$ treatment for $12 \mathrm{~h}$, followed by $2.5 \mu \mathrm{M}$ or $5 \mu \mathrm{M}$ Nerol for $12 \mathrm{~h}$, followed by CCK-8 assay. E. H9c2 cardiomyocytes were pre-treated with H/R treatment for $12 \mathrm{~h}$, followed by $2.5 \mu \mathrm{M}$ or $5 \mu \mathrm{M}$ Nerol for $12 \mathrm{~h}$, followed by LDH release assay. $\mathrm{n}=8 . \mathrm{p}<0.05\left(^{*}\right), \mathrm{p}<0.01\left({ }^{* *}\right)$.

\section{Western blot analysis}

The total protein concentrations in the whole cell lysate were measured by using the Bicinchoninic Acid (BCA) Protein Assay Kit (Thermo Scientific, Rockford, IL) and 20-30 $\mu \mathrm{g}$ protein aliquots were denatured by heating at $95 \mathrm{oC}$ for $10 \mathrm{~min}$ in sodium dodecyl sulfate (SDS) loading buffer. Protein was separated on a $12 \%$ SDSpolyacrylamide gel electrophoresis (PAGE) gel and then transferred to polyvinylidene difluoride membranes. The following primary antibody were used: poly (ADPribose) polymerase (PARP); cleaved PARP; MCL-1; Bim; phosphor-p85 (p-p85); PI3K p85; phosphor-AKT (p-AKT); total-AKT; phosphor-p38 (p-p38); mitogenactivated protein kinase (MAPK) p38; and glyceraldehyde 3-phosphate dehydrogenase (GAPDH). Antibodies were provided by Cell Signaling. The relative protein expression levels were normalized to GAPDH by ImageJ software.

\section{Caspase- 3 activity assay}

To evaluate the activity of caspase-3 in H9c2 cells with different treatments, a colorimetric caspase-3 activity assay kit (Beyotime) was used according to the manufacture's instruction. A microplate reader was used to read the absorbance at a wavelength of $485 \mathrm{~nm}$.

\section{Statistical analysis}

The data were presented as mean \pm standard deviation (SD), which was confirmed by at least three independent experiments. One-way analysis of variance (ANOVA) models with Tukey's post-hoc tests were used. P value less than 0.05 was considered statistically significant.

\section{Results}

Nerol ameliorated H/R-induced injury in $\mathrm{H} 9 \mathrm{c} 2$ cells in a dose-dependent manner

Comparing to the cells cultured in normoxic conditions, $\mathrm{H} 2 \mathrm{c} 9$ cells had significantly lower cell viability and higher LDH release after H/R induction (Figure 1B \& C). In contrast, the cell viability was promoted while LDH release was inhibited by the treatment with Nerol, respectively (Figure 1B \& C). Additionally, the cell 
A

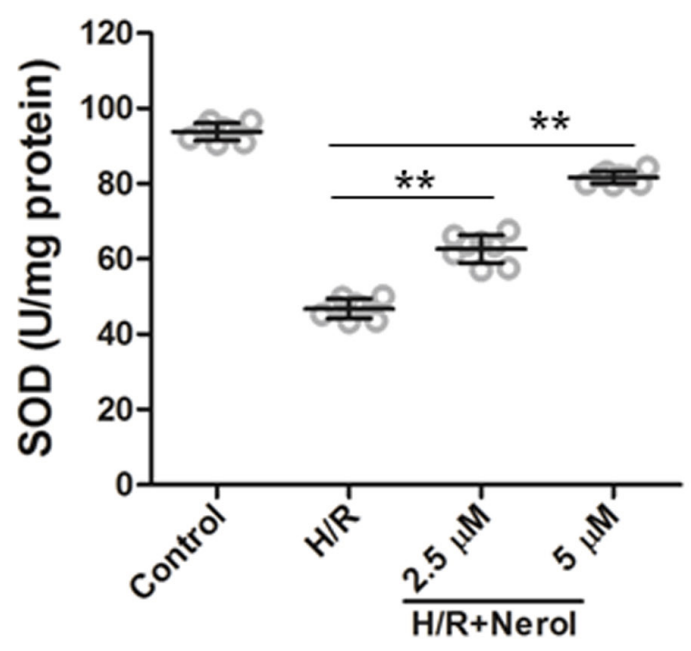

C

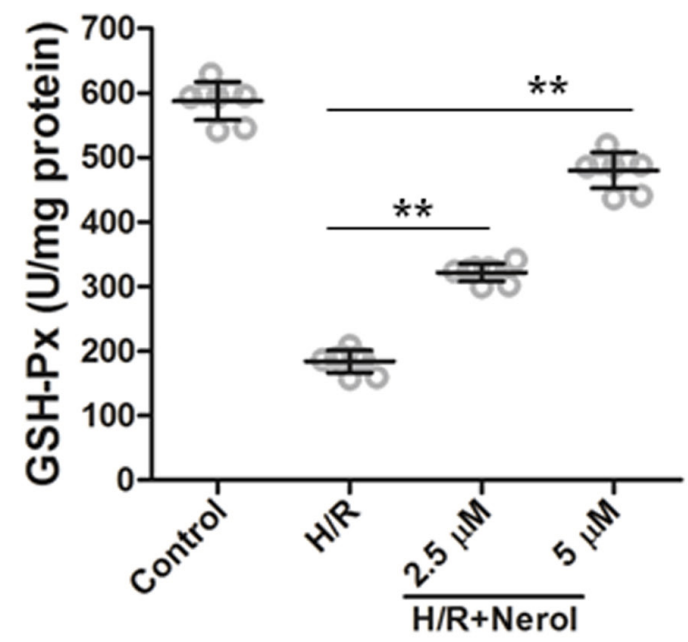

B

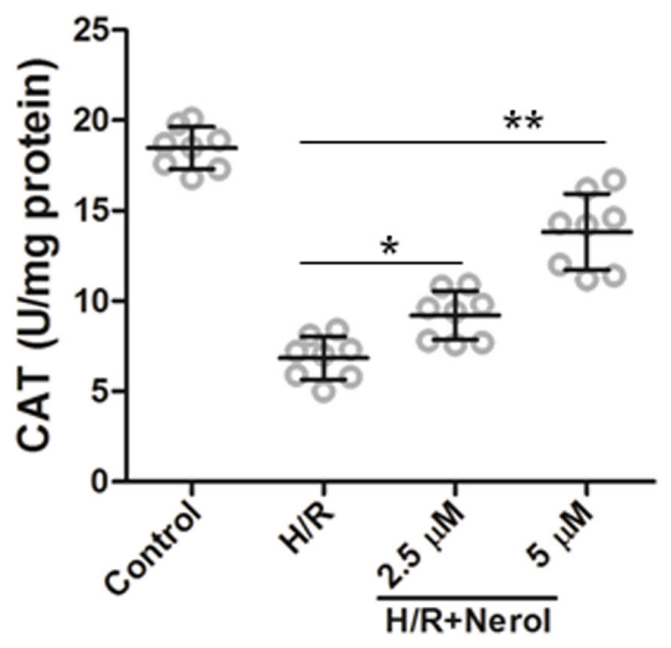

D

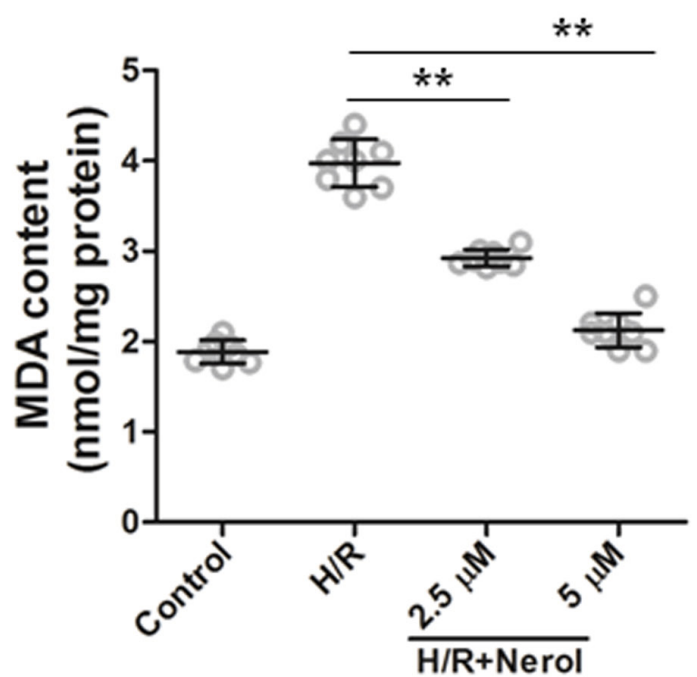

Figure 2. Nerol suppresses H/R-induced oxidative stress in cardiomyocytes. H9c2 cardiomyocytes were treated with $2.5 \mu \mathrm{M}$ or 5 $\mu \mathrm{M}$ Nerol, followed by H/R treatment for $12 / 12 \mathrm{~h}$. The activities of SOD (A), CAT (B), GSH-Px (C) and MDA content (D) were measured in $\mathrm{H} 9 \mathrm{c} 2$ cardiomyocytes. $\left.\mathrm{n}=8 . \mathrm{p}<0.05\left(^{*}\right), \mathrm{p}<0.01{ }^{* *}\right)$.

viability increased and LDH release decreased with increasing Nerol concentration (Figure 1B \& C), indicating the effects of Nerol on cell viability and LDH release exhibited a dose-dependent manner. The above results showed that Nerol displayed protective effects against H/R injury. Moreover, we also examined the inhibitory effects of Nerol by treating the cells after $\mathrm{H} / \mathrm{R}$-induced injury, and found that cell viability and LDH release were significantly promoted and inhibited, respectively (Figure $1 \mathbf{D} \boldsymbol{\&} \mathbf{E}$ ). These results revealed the protective and inhibitory effects of Nerol against pre- and post-H/R-induced injuries, respectively.

Nerol ameliorated H/R-induced oxidative stress injury in $\mathrm{H} 9 \mathrm{c} 2$ cells in a dose-dependent manner

To investigate the effect of Nerol on H/R induced oxidative in $\mathrm{H} 9 \mathrm{c} 2$ cells, the activities of oxidative stress markers were measured. The levels of SOD, CAT and
GSH-Px were markely decreased compared to the cells cultured under normoxic conditions; however, the levels of these markers in the cells with Nerol treatments were notably higher than the H/R stimulation (Figure 2A, B, $\boldsymbol{\&}$ C). With Nerol concentration increased, the activities of Nerol on the anti-oxidant enzyme also increased. Interestingly, H/R stimulation remarkably promoted MDA content, which could be inhibited by the application of Nerol regardless of concentrations (Figure 2D). The effect of Nerol on MDA production also exhibited a dosedependent manner and MDA content decreased with increasing Nerol concentration (Figure 2D).

\section{Nerol inhibited cell apoptosis in a dose-dependent manner}

To evaluate the effects of Nerol on H9c2 cell apoptosis after processed H/R treatment, flow cytometry analysis was used. Compared to the control, the H/R-treated cells showed a significantly higher apoptotic rate; however, 
A

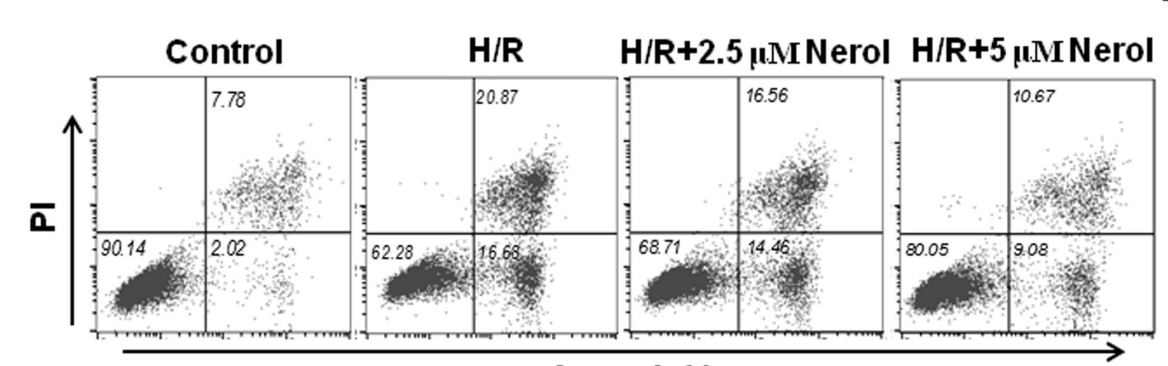

Annexin V

C

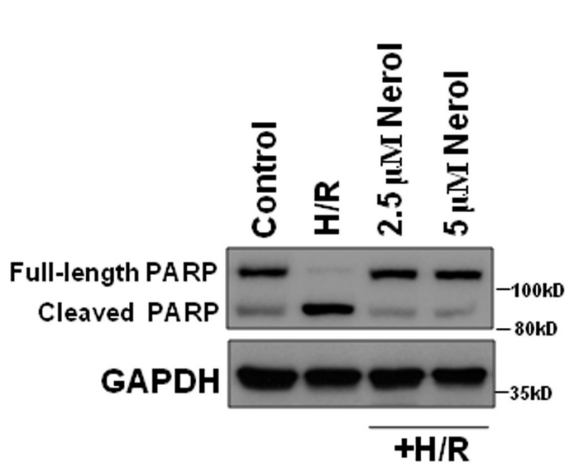

B

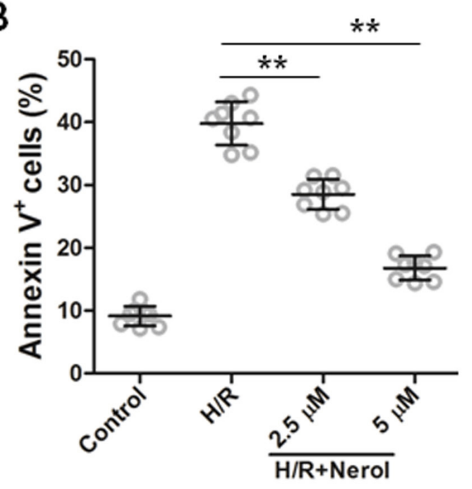

$\mathbf{E}$

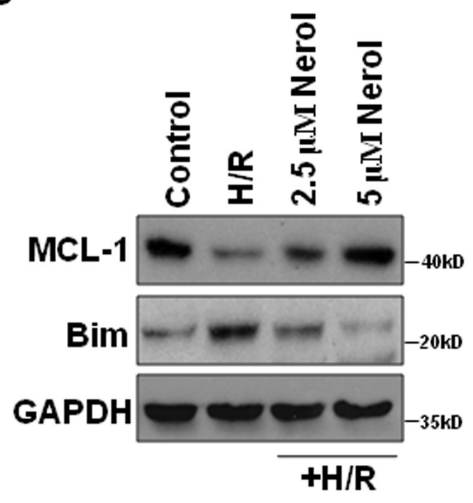

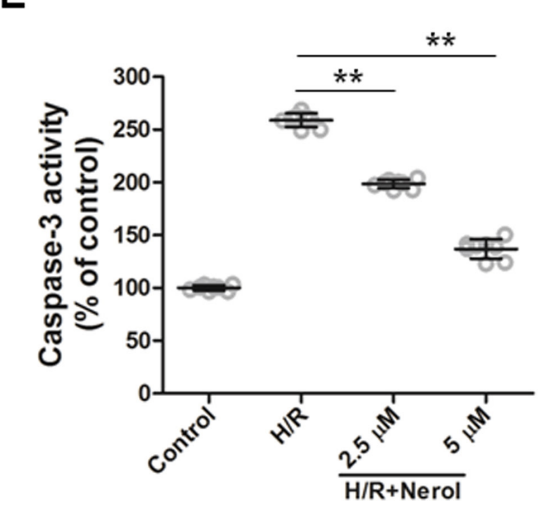

Figure 3. Nerol inhibited H/R-induced apoptosis in cardiomyocytes. (A \& B) H9c2 cardiomyocytes were treated with $2.5 \mu \mathrm{M}$ or 5 $\mu \mathrm{M}$ Nerol, followed by $\mathrm{H} / \mathrm{R}$ treatment for $12 / 12 \mathrm{~h}$. And then cells were stained with Annexin V-FITC and PI, followed by cell apoptosis analysis on a flow cytometer (A). Percentages of annexin V+ are indicated (B). (C) H9c2 cardiomyocytes were treated with $2.5 \mu \mathrm{M}$ or 5 $\mu \mathrm{M}$ Nerol, followed by H/R treatment for $12 / 12 \mathrm{~h}$. Then, cells were lysed for western blot against PARP, a biomarker of cell apoptosis. (D) The above cells were also lysed for western blot against MCL-1 and Bim. GAPDH was used as a loading control. E. The activity of caspase-3 in treated H9c2 cardiomyocytes was examined by caspase- 3 activity assays. $\left.n=8 . p<0.01{ }^{* *}\right)$.

Nerol decreased apoptotic rate regardless of its concentrations (Figure $\mathbf{3 A} \& \mathbf{B}$ ). Meanwhile, the expression of cleaved PARP and Bim was enhanced due to $\mathrm{H} / \mathrm{R}$ induction; while the expression of full-length PARP and MCL-1 was abolished (Figure 3C \& D). By contrast, the application of Nerol significantly suppressed the protein levels of cleaved PARP and Bim, and promoted the expression of full-length PARP and MCL-1 in a dose-dependent manner (Figure 3C \& D), suggesting Nerol alleviated the H/R-induced apoptotic protein expression and inhibited the cleavage of PARP. To further confirm the inhibitory effect of Nerol on H/R-induced apoptosis in H9c2 cells, the activity of caspase-3 was quantified. The results showed that the H/R stimulation significantly promoted the caspase-3 activity, which could be suppressed by Nerol (Figure 3E). Collectively, with the Nerol concentration increased, the cell apoptosis rate, the apoptotic protein expression (cleaved PARP and Bim), and the caspase- 3 activity also decreased. These results indicated that Nerol ameliorated H/R-induced cell apoptosis in $\mathrm{H} 9 \mathrm{c} 2$ cells in a dose-dependent manner.

Nerol upregulated PI3k/AKT and MAPK signaling pathways in $\mathrm{H9c} 2$ cells

Compared to the control cells, H/R stimulation significantly inhibited PI3K p85, AKT and MAPK p38 phosphorylation (Figure 4A-F). As shown in Figure 4, the phosphorylation of PI3K p85, AKT and MAPK p38 was remarkably upregulated in response to Nerol treatment regardless of the concentrations. These above results indicated that Nerol upregulated PI3K/AKT and MAPK signaling pathways in $\mathrm{H} / \mathrm{R}$-stimulated $\mathrm{H} 9 \mathrm{c} 2$ cells.

\section{Nerol inhibited H/R-induced injury by activation of $\mathrm{PI3K}$ in $\mathrm{H9c2}$ cells}

To evaluate whether the cardioprotective effects of Nerol on $\mathrm{H} 9 \mathrm{c} 2$ cells were mediated via the PI3K/AKT signaling pathway, the cell viability and LDH release were measured after treatment of PI3K inhibitors (BEZ235 or LY294002). There was no significant differences between the cells treated with Nerol and the control in terms of cell viability and LDH release (Figure 5). In contrast, the cells treated with both Nerol and BEZ235 displayed significantly lower cell viability and greater $\mathrm{LDH}$ release after $\mathrm{H} / \mathrm{R}$ stimulation than the cells treated with Nerol alone (Figure 5 A \& B). Besides, the cell viability and LDH release in cells treated with both Nerol and LY294002 were also restored (Figure 5C \& D). Collectively, these results indicated that Nerol alleviated $\mathrm{H} / \mathrm{R}$-induced injury through activating $\mathrm{PI} 3 \mathrm{~K}$ in H9c2 cells. 
A

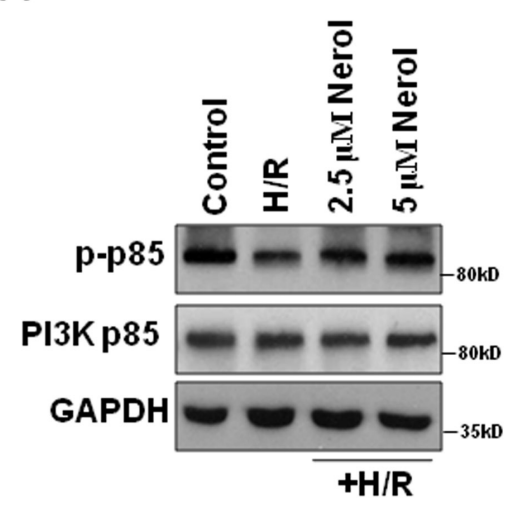

D

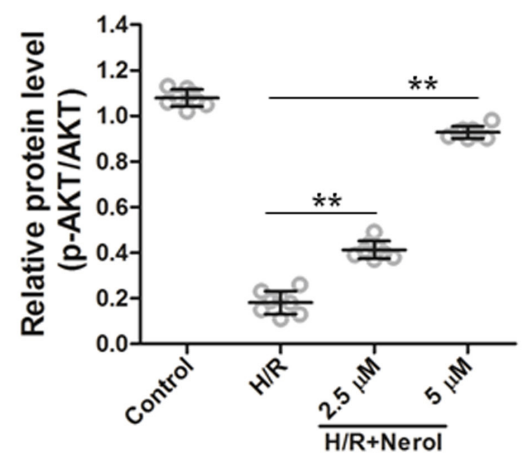

B

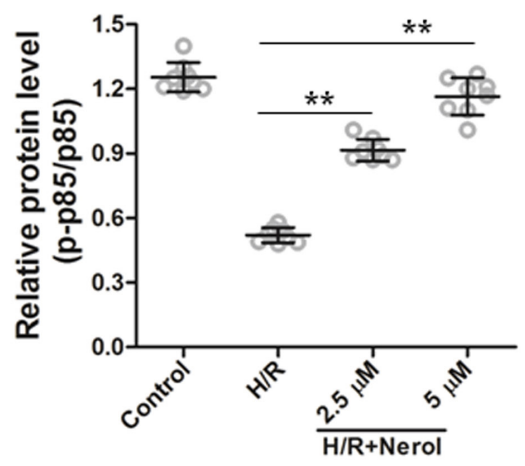

E

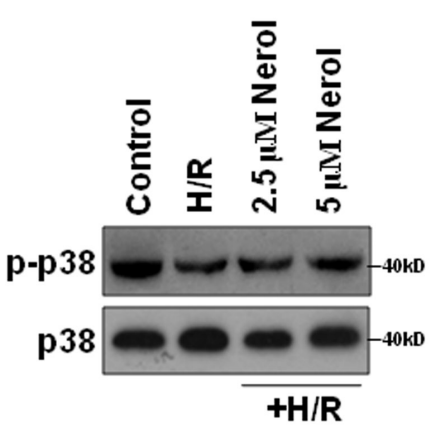

C

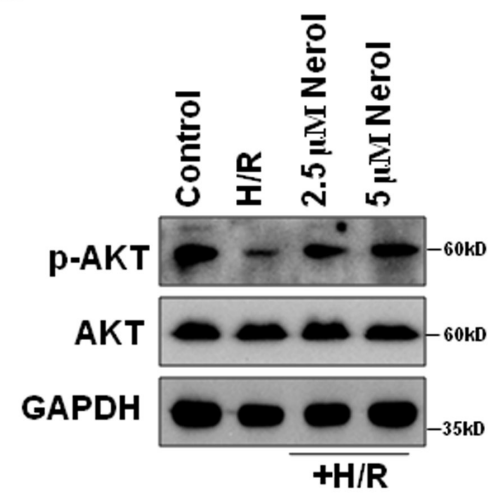

Figure 4. Nerol upregulated PI3K/AKT signaling and MAPK signaling in cardiomyocytes. (A \& B) H9c2 cardiomyocytes were treated with $2.5 \mu \mathrm{M}$ or $5 \mu \mathrm{M}$ Nerol, followed by H/R treatment for $12 / 12 \mathrm{~h}$. Then, cells were lysed for western blot against p-p85 and p85 (A). Optical density analysis for p-p85 was indicated (B). (C \& D) H9c2 cardiomyocytes were treated with $2.5 \mu \mathrm{M}$ or $5 \mu \mathrm{M}$ Nerol, followed by $\mathrm{H} / \mathrm{R}$ treatment for $12 / 12 \mathrm{~h}$. Then, cells were lysed for western blot against $\mathrm{p}-\mathrm{AKT}$ and AKT (C). Optical density analysis for $\mathrm{p}-\mathrm{AKT}$ was indicated (D). (E \& F) H9c2 cardiomyocytes were treated with $2.5 \mu \mathrm{M}$ or $5 \mu \mathrm{M}$ Nerol, followed by $\mathrm{H} / \mathrm{R}$ treatment for $12 / 12 \mathrm{~h}$. Then, cells were lysed for western blot against p-p38 and p38 (E). Optical density analysis for p-p38 was indicated $(F) . n=8 .{ }^{* *} p<0.01\left({ }^{* *}\right)$.

\section{Discussion}

This study indicated that Nerol protected cardiomyocytes against $\mathrm{H} / \mathrm{R}$-induced injuries, during which the PI3K/AKT signaling pathway might be involved. This was evidenced by increased cell viability, oxidative stress marker activities (SOD, CAT, and GSH-Px) and Bcl-2 family protein expression. Moreover, the decreased LDH release, the expression of cell-death proteases, and the increased caspase- 3 activity also confirmed our findings. Finally, we found that the inhibiting PI3K/AKT activation diminished the protective effects of Nerol.

The in vitro $\mathrm{H} / \mathrm{R}$ model has been widely used to mimic several cardiac pathological situations, and it is easy to control the physical and chemical environment in the H/R model (13). Mounting evidence demonstrates that $\mathrm{H} / \mathrm{R}$ is sufficient to cause injuries in various cell types through in vitro experiments. Thus, cellular models of $\mathrm{H} / \mathrm{R}$ are useful tools to evaluate the potent cardiac protective effects of novel agents (14). Both necrosis and apoptosis are associated with the microenvironment homeostasis disrupted by cellular hypoxia and linked to excess intracellular ROS production $(14,15)$. Herein, we revealed that cell viability was inhibited, $\mathrm{LDH}$ release was elevated, oxidative stress markers were upregulated, and caspase-3 activity was enhanced upon $\mathrm{H} / \mathrm{R}$ stimulation in $\mathrm{H} 9 \mathrm{c} 2$ cells, suggesting that $\mathrm{H} / \mathrm{R}$ can indeed induce myocardial injury in vitro.

Nerol, an acyclic monoterpene alcohol, has been studied as an anti-oxidant and free radical scavenger (9). Previous studies reveal that Nerol significantly promoted cell viability, SOD activity and glutathione content in preoxide stressed rat alveolar macrophages; meanwhile, the lipid peroxidation, nitrogen oxide release and ROS generation were suppressed (11). To confirm the inhibitory effects of Nerol on cell apoptosis and necrosis, the expression of hallmark apoptotic and necrotic proteins was analyzed in our present study. The activity of caspases 3 cleaves PARP in fragments of 89 and $24 \mathrm{kDa}$ during apoptosis (16). The function of full-length PARP is to repair DNA damage in response to a variety of cellular stresses via adding poly (ADP ribose) polymers (17). Moreover, caspase- 3 is considered as one of the executor caspases in apoptosis and initiates apoptotic DNA fragmentation (18). In our current study, the activities of caspase 3 and cleaved PARP were enhanced, indicating cell apoptosis upon $H / R$ stimulation. In contrast, the expression of cleaved PARP and the caspase 3 activity were restored in the Nerol-pretreated cells, indicating that Nerol may play an important role in protecting DNA against the oxidative stress-induced damage. The effects 
A

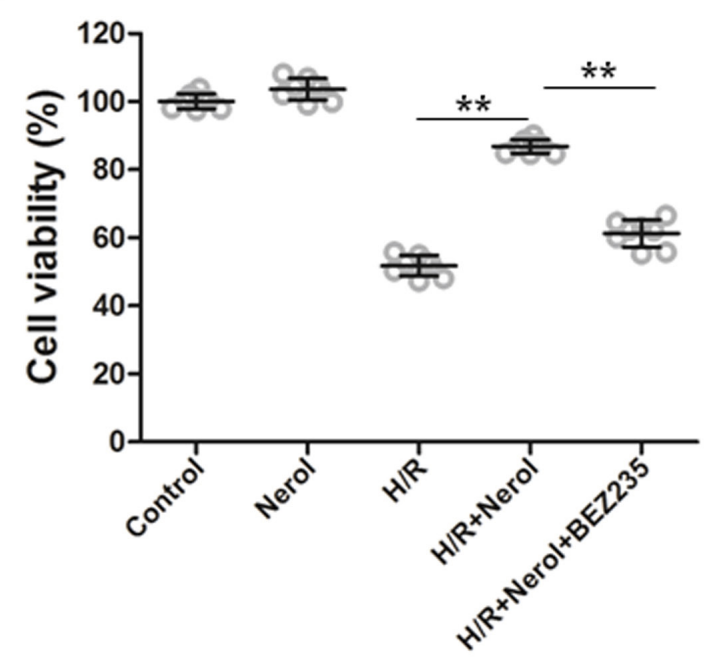

C

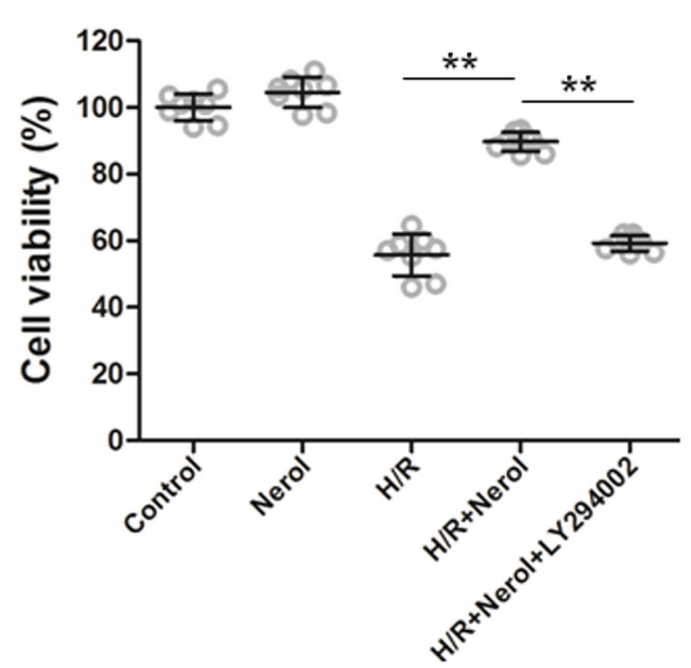

B

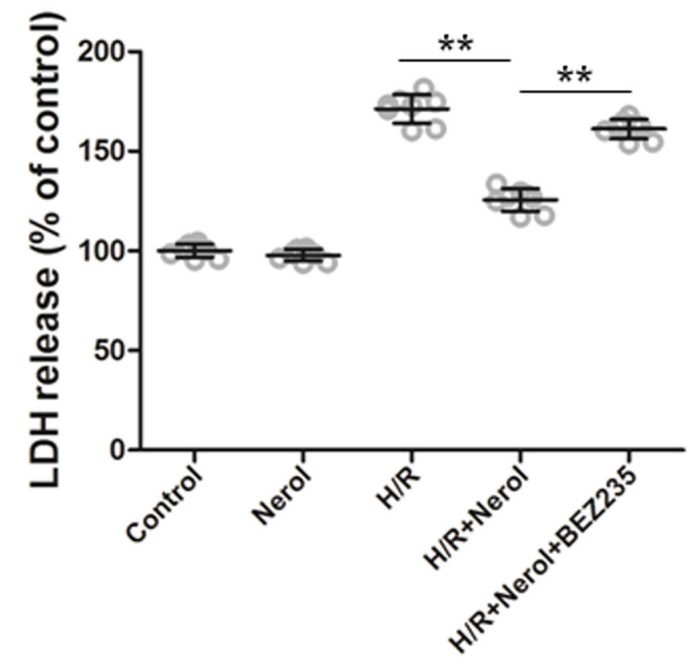

D

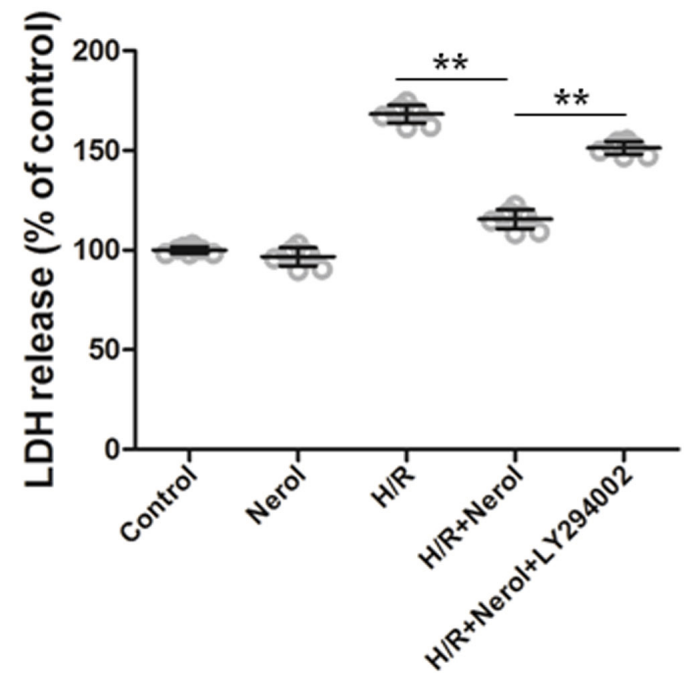

Figure 5. The effects of Nerol were abolished by the addition of PI3K inhibitors in cardiomyocytes. (A \& B) H9c2 cardiomyocytes were treated with $5 \mu \mathrm{M} \mathrm{Nerol}$ in the presence or absence of $5 \mu \mathrm{M} \mathrm{BEZ235}$, followed by H/R treatment for $12 / 12 \mathrm{~h}$, or H9c2 cardiomyocytes were only treated with $5 \mu \mathrm{M}$ Nerol for $24 \mathrm{~h}$. Then, cell viability was evaluated by CCK-8 assay (A), and LDH release was estimated by LDH release assay (B). (C \& D) H9c2 cardiomyocytes were treated with $5 \mu \mathrm{M}$ Nerol in the presence or absence of $5 \mu \mathrm{M}$ LY294002, followed by H/R treatment for $12 / 12 \mathrm{~h}$, or H9c2 cardiomyocytes were only treated with $5 \mu \mathrm{M}$ Nerol for $24 \mathrm{~h}$. Then, cell viability was evaluated by CCK-8 assay (C), and LDH release was estimated by LDH release assay (D). $n=8 . p<0.01\left(^{* *}\right)$.

of Nerol on the expression of MCL-1, an anti-apoptotic Bcl-2 family protein, confirmed these findings. In a cardiomyocyte-specific MCL-1 knockout mouse model, the progression of heart failure was delayed and the survival was extended (19). Silencing Bim was also shown to significantly attenuate oxygen deprivation-induced apoptosis in $\mathrm{H} 9 \mathrm{c} 2$ cardiomyocytes (20). The expression of Bim in the H9c2 cells with Nerol pretreatment was diminished, suggesting that Nerol may inhibit cell apoptosis via negatively regulating Bim degradation. The findings of our study are consistent with these earlier reports and may suggest that the pretreatment with Nerol can significantly protect cardiomyocytes from $\mathrm{H} / \mathrm{R}$-induced apoptosis via mediating apoptotic protein expression.

To explore the potential mechanisms underlying Nerol mediated $\mathrm{H} 9 \mathrm{c} 2$ cell apoptosis, the PI3K/AKT and
MAPK signaling pathways were evaluated. In various cell systems, activation of PI3K/AKT stimulates cell proliferation, growth and survival. In the heart, due to the limited proliferative capacity, this pathway plays a critical role in regulating cardiomyocyte growth and survival $(21,22)$. PI3K is composed of a catalytic subunit p110 and a regulatory subunit p85. Increasing evidence has demonstrated that $\mathrm{p} 85$ can mediate PI3K signal down-regulation via binding to 3-poly-Ptdlns or Scr homology region 2 domain-containing phosphatase-1 (SHP-1) phosphatase (23). AKT is the key downstream effector of PI3K in regulating cardiac growth (21). In our current study, we found that after exposed to Nerol, the phosphorylation of AKT and p 85 was significantly promoted, indicating that the upregulated phosphorylation of AKT and p85 is associated with cell apoptosis and 
viability. In numerous systems, the activation of PI3K/ AKT can provide pro-survival signals to prevent cell apoptosis. Constitutively activating PI3K or AKT can reduce hypoxia-induced apoptosis in vitro and cause remarkably reduced cardiomyocyte apoptosis and infarct size in vivo (24) Moreover, the cardiac function and morphology can be preserved both in vitro and in vivo as a result of AKT activation (22) Taken together, our findings suggested that the application of Nerol might be beneficial in cardiomyocytes in vitro through activating the PI3K/ AKT signaling pathway. Interestingly, we also found that the phosphorylation of MAPK p38 was enhanced following Nerol pretreatment compared to the H/R control without pretreatment, indicating that the MAPK signaling pathway may also be involved. However, the detailed interaction between Nerol and the MAPK signaling pathway and the potential mechanisms are still elusive. In our further investigation, the effects of Nerol on the MAPK signaling will be studied.

To confirm the role of PI3K/AKT signaling pathway on the Nerol-mediated cardiac protection, BEZ235 and LY294002, two specific PI3K inhibitors, were used in the current study. The enhanced cell viability and LDH release in the Nerol pretreated cells were abolished following the addition of BEZ235 or LY294002. The PI3K/AKT/mTOR signaling cascade plays a critical role in cell proliferation, survival and metabolism. The core components of the $\mathrm{PI} 3 \mathrm{~K} / \mathrm{AKT} / \mathrm{mTOR}$ cascade are composed of PI3K and its downstream mediators AKT and mTOR (25). BEZ235 is a dual PI3K/mTOR inhibitor and inhibits both PI3K and mTOR kinase activities via binding to the adenosine triphosphate (ATP)-binding sites of PI3K and mTOR (26). Additionally, BEZ235 can inhibit several downstream effectors, such as AKT, ribosomal protein S6 and translation initiation factor 4E binding protein 1 (27). To explore the target signaling pathway, LY294002, a reversible inhibitor of $\mathrm{PI} 3 \mathrm{~K}$ and $\mathrm{mTOR}$, was used to treat H9c2 cells. Similarly, the elevated cell viability and LDH release from the pretreatment of Nerol was abolished, further confirming that Nerol ameliorated the H/R-induced injuries via activating the PI3K/AKT signaling pathway. However, some limitations existed in the current study. Firstly, previous studies revealed that distinct potassium channels protected cardiac injuries via interacting with the PI3K/AKT signaling pathway $(22,28)$. The genetic loss of tandem pore domain acid sensitive $\mathrm{K}+$ (TASK1) channel can preserve cardiac function in a pressure overload model, which is associated with the AKT phosphorylation augmentation (22). Our current study demonstrated that both PI3K/AKT and MAPK signaling pathways were involved in the protective effects of Nerol on cardiomyocytes. Additionally, selectively inhibiting the PI3K signaling pathway can reverse the alleviating effects of Nerol; however, the detailed molecular mechanisms are elusive. Furthermore, the impact of Nerol on the MAPK signaling pathway is elusive. Therefore, in our further investigation, it is necessary to determine the potential direct targets of Nerol. Secondly, H9c2 cells were employed in the present study, and the in vitro protective effects were revealed; thus, the potential in vivo cardio-protective effects of Nerol are warranted in further investigation. Thirdly, the H9c2 cell line is a rat embryonic cardiomyocyte cell line, and primary cardiomyocytes are more relevant as they are closer to physiological conditions. Therefore, we will use primary cardiomyocytes for our further investigation to confirm our current findings. It should also be noted that there was no positive control in our experiments, which should be included in future studies.

\section{Conclusion}

In summary, we hereby report that Nerol alleviates $\mathrm{H} / \mathrm{R}$-induced injuries in $\mathrm{H} 9 \mathrm{c} 2$ cells by promoting cell viability, attenuating apoptosis and oxidative stress via activating the PI3K/AKT signaling pathway. Therefore, our study suggests that Nerol could be used as a potential therapeutic strategy for $\mathrm{H} / \mathrm{R}$-induced injuries.

\section{Conflict of interest}

The authors declare that they have no conflicts of interest to disclose.

\section{References}

1. Essop MF. Cardiac metabolic adaptations in response to chronic hypoxia. J Physiol. 2007; 584:715-726.

2. Samaja M, Milano G. Editorial-Hypoxia and reoxygenation: from basic science to bedside. Front Pediatr. 2015; 3:86.

3. Morales CR, Pedrozo Z, Lavandero S, Hill JA. Oxidative stress and autophagy in cardiovascular homeostasis. Antioxid Redox Signal. 2014; 20:507-518.

4. Farías JG, Molina VM, Carrasco RA, Zepeda AB, Figueroa $E$, Letelier $P$, et al. Antioxidant therapeutic strategies for cardiovascular conditions associated with oxidative stress. Nutrients 2017; 9:966.

5. Zeng Y, Song JX, Shen XC. Herbal remedies supply a novel prospect for the treatment of atherosclerosis: A review of current mechanism studies. Phytother Res. 2012; 26:159-167.

6. Bagchi D, Sen CK, Ray SD, Das DK, Bagchi M, Preuss HG, et al. Molecular mechanisms of cardioprotection by a novel grape seed proanthocyanidin extract. Mutat Res. 2003; 523:87-97

7. Guillermo Gormaz J, Quintremil S, Rodrigo R. Cardiovascular disease: a target for the pharmacological effects of quercetin. Curr Top Med Chem. 2015; 15:1735-1742.

8. Fathima SN, Murthy SV. Cardioprotective effects to chronic administration of Rosa Damascena petals in isoproterenol induced myocardial infarction: biochemical, histopathological and ultrastructural studies. Biomed Pharmacol J. 2019; 12:1155-1166.

9. Chen W, Viljoen AM. Geraniol - A review of a commercially important fragrance material. South African Journal of Botany 2010; 76:643-651.

10. Mileva M, Krumova E, Miteva-Staleva J, Kostadinova N, Dobreva A, Galabov AS. Chemical compounds, in vitro antioxidant and antifungal activities of some plant essential oils belonging to rosaceae family. Comptes rendus de l'Académie bulgare des Sciences 2014; 67.

11. Tiwari M, Kakkar P. Plant derived antioxidants-geraniol and camphene protect rat alveolar macrophages against t-BHP induced oxidative stress. Toxicol In Vitro. 2009; 23:295-301.

12. Menezes-Filho JERd, Souza DSd, Santos-Miranda A, Cabral VM, Santos JNA, Cruz JdS, et al. Nerol attenuates ouabain- 
induced arrhythmias. Evid Based Complement Alternat Med. 2019; 2019:5935921..

13. Portal L, Martin V, Assaly R, d'Anglemont de Tassigny A, Michineau S, Berdeaux A, et al. A model of hypoxiareoxygenation on isolated adult mouse cardiomyocytes: characterization, comparison with ischemia-reperfusion, and application to the cardioprotective effect of regular treadmill exercise. J Cardiovasc Pharmacol Ther. 2013; 18:367-375.

14. Li C, Jackson RM. Reactive species mechanisms of cellular hypoxia-reoxygenation injury. American Journal of Physiology-Cell Physiology 2002; 282:C227-C241.

15. Konstantinidis K, Whelan RS, Kitsis RN. Mechanisms of cell death in heart disease. AAm J Physiol Cell Physiol. 2012; 32:1552-1562.

16. Kaufmann SH, Desnoyers S, Ottaviano Y, Davidson NE, Poirier GG. Specific proteolytic cleavage of poly (ADP-ribose) polymerase: an early marker of chemotherapy-induced apoptosis. Cancer Res. 1993; 53:3976-3985.

17. Chaitanya GV, Alexander JS, Babu PP. PARP-1 cleavage fragments: signatures of cell-death proteases in neurodegeneration. Cell Commun Signal. 2010; 8:31.

18. Mcllwain DR, Berger T, Mak TW. Caspase functions in cel death and disease. Cold Spring Harb Perspect Biol. 2015; 7.

19. Thomas RL, Roberts DJ, Kubli DA, Lee Y, Quinsay MN, Owens JB, et al. Loss of MCL-1 leads to impaired autophagy and rapid development of heart failure. Genes Dev. 2013; 27:1365-1377.

20. $X u$ Y, Xing $Y, X u$ Y, Huang $C$, Bao $H$, Hong $K$, et al. Pim-2 protects $\mathrm{H} 9 \mathrm{c} 2$ cardiomyocytes from hypoxia/reoxygenationinduced apoptosis via downregulation of Bim expression. Environ Toxicol Pharmacol. 2016; 48:94-102.

21. Matsui T, Nagoshi T, Rosenzweig A. Akt and PI 3-kinase signaling in cardiomyocyte hypertrophy and survival. Cell Cycle 2003; 2:219-222.

22. Duan W, Hicks J, Makara MA, Ilkayeva O, Abraham DM. TASK-1 and TASK-3 channels modulate pressure overloadinduced cardiac remodeling and dysfunction. Am J Physiol Heart Circ Physiol. 2020; 318:H566-H580.

23. Jiménez C, Hernández C, Pimentel B, Carrera AC. The p85 regulatory subunit controls sequential activation of phosphoinositide 3-kinase by Tyr kinases and Ras. J Biol Chem. 2002; 277:41556-41562.

24. Matsui T, Tao J, del Monte F, Lee K-H, Li L, Picard M, et al. Akt activation preserves cardiac function and prevents injury after transient cardiac ischemia in vivo. Circulation 2001; 104:330-335.

25. Yu JSL, Cui W. Proliferation, survival and metabolism: the role of PI3K/AKT/mTOR signalling in pluripotency and cell fate determination. Development 2016; 143:3050.

26. Gobin B, Battaglia S, Lanel R, Chesneau J, Amiaud J, Rédini $F$, et al. NVP-BEZ235, a dual PI3K/mTOR inhibitor, inhibits osteosarcoma cell proliferation and tumor development in vivo with an improved survival rate. Cancer Lett. 2014; 344:291-298.

27. Durrant DE, Das A, Dyer S, Tavallai $S$, Dent P, Kukreja RC. Targeted inhibition of phosphoinositide 3-kinase/mammalian target of rapamycin sensitizes pancreatic cancer cells to doxorubicin without exacerbating cardiac toxicity. Mol Pharmacol. 2015; 88:512-523.

28. Abraham DM, Lee TE, Watson LJ, Mao L, Chandok G, Wang $\mathrm{H}-\mathrm{G}$, et al. The two-pore domain potassium channel TREK-1 mediates cardiac fibrosis and diastolic dysfunction. J Clin Invest. 2018; 128:4843-4855. 